\title{
LV-PP-3-4
}

\section{Prognostic significance of the serosal invasion in hepatocellular carcinoma}

\author{
Incheon KANG ${ }^{1}$, Dai Hoon HAN*2 \\ 'Department of Surgery, CHA Bundang Medical Center, CHA University School of Medicine, Seongnam, Korea \\ ${ }^{2}$ Department of Surgery, Yonsei University College of Medicine, Seoul, Korea
}

Introduction: The prognostic significance of serosal invasion (SI) was not clarified in patients with hepatocellular carcinoma (HCC). We investigated the impact of SI for the long-term outcome after curative surgical resection in patients with HCC.

Methods: In total, 783 consecutive patients with HCC who underwent curative surgical resection without any preoperative treatment were histologically evaluated for SI. Patients with SI were classified into two groups: positive for SI (pSI; $\mathrm{n}=333$ ) and negative for SI (nSI; $\mathrm{n}=450$ ). Clinicopathologic features, disease-free survival (DFS), and overall survival (OS) were compared between patients with pSI and nSI.

Results: The pSI group showed more aggressive tumor characteristics, such as higher tumor marker levels (alpha-fetoprotein, $p=$ 0.023 ; protein induced by vitamin K absence-II, $p=0.016)$, larger tumor size $(p<0.001)$, higher prevalence of microvascular invasion $(p$ $=0.002)$, and poorer differentiation $(p=0.002)$, than the nSI group. In survival analysis, 5 -year DFS was $48.1 \%, 61.2 \%$ in the pSI, nSI, respectively $(p<0.001)$, while 5 -year OS was $74.8 \%, 86.1 \%$, respectively $(p=0.002)$. In multivariate analysis, SI was an independent prognostic factor for DFS $(p=0.043)$ and OS $(p=0.042)$.

Conclusions: The SI was associated with more aggressive clinicopathologic characteristics and a higher risk of poor survival. Therefore, intensive careful follow-up is essential for patients with pSI after curative surgical resection in patients with HCC. 